\title{
Autism and adult attachment: Issues with current measurement practices
}

Abi M. B. Davis \& Robin S. S. Kramer

School of Psychology, University of Lincoln, Lincoln, UK

Correspondence: Abi M. B. Davis, School of Psychology, University of Lincoln, Brayford Pool, Lincoln LN6 7TS, UK.

E-mail: abigailmarydavis@gmail.com

Telephone: +44 (0)1522 882000

Fax: $+44(0) 1522886489$

This is a pre-print with copyright owned by the authors.

Running title: autism and adult attachment 


\begin{abstract}
Attachment styles in individuals with autism are not well understood, and research into the topic is limited to date. Authors regularly utilise standardised measures to classify attachment in adulthood, and this is the case for research with neurotypical and autistic populations. Here, we argue that there may be fundamental problems with using such measures, developed for neurotypical populations, in order to quantify attachment in those with autism. Crucially, such tools may be unable to differentiate between autistic behaviours and behaviours associated with insecure attachment styles. Furthermore, many studies which have investigated attachment and autism may lack sufficient statistical power due to the use of time-consuming attachment interviews or student populations which typically do not contain sufficient numbers of adults with autism. We argue that it is essential that measures are developed which accurately distinguish between insecure attachment styles and behaviours associated with autism, with the goal of better understanding attachment in those with autism for both parental and romantic relationships.
\end{abstract}

Keywords: autism spectrum disorder; attachment; questionnaire; interview; measurement 


\section{Introduction}

Given the importance of attachment for long-term social and romantic outcomes in adulthood (Beffel et al., 2020), it is crucial that we attempt to understand factors that may influence its development. Although few studies have explored autism and attachment, research suggests that children with autism are at a greater risk of developing insecure attachments than neurotypical children (Rutgers et al., 2004), and that this remains the case for autistic adults in their romantic relationships (Lau \& Peterson, 2011). Typically, this conclusion relies on assessments of attachment such as the Strange Situation in childhood or self-report questionnaires in adulthood.

Problematically, the latter method of assessment in particular may be unable to differentiate between autistic traits and those associated with insecure attachment styles. Posed as a question, are characteristics used to categorise attachment style displayed by autistic people due to their autism rather than their attachment style? If so, there would be an immediate need to revise current measures before any conclusions can be drawn regarding autism and attachment.

\section{Attachment questionnaires and autism}

Previous research (e.g., Lau \& Peterson, 2011) has consistently shown that "avoidant" attachment styles are commonly assigned when evaluating autistic adults' romantic relationships. Typically, authors use self-report tools such as the Experiences in Close Relationship Scale (ECR; Brennan et al., 1998) to assess adult attachment styles. However, these instruments were developed for use with neurotypical adults. By applying the same tool for use with an autistic sample, researchers fail to account for the specific social and 
communication difficulties which may be exhibited in both those with insecure attachments and those with autism. Although much research has acknowledged the association between higher Autism Spectrum Quotient (AQ; Baron-Cohen et al., 2001) scores and insecure attachment behaviours, authors should be cautious when ascribing causation. This is due to the potential for behaviours in those with autism and those with insecure attachments (particularly "avoidant”) to closely mirror each other. To illustrate, a neurotypical, insecurely attached adult may strongly agree with the statement "I get uncomfortable when a romantic partner wants to be very close" (an ECR item) because they fear intimacy due to issues surrounding trust. In contrast, an adult with autism may respond similarly due to their very different physical sensory issues. To consider another example, disagreement with the statement "I am confident that other people will like and respect me" (taken from the Attachment Style Questionnaire; Feeney et al., 1994) is attributed to an "anxious/preoccupied" attachment style, and is purported to be indicative of insecure attachment to a romantic partner. For an autistic adult, this may be a simple matter-of-fact acknowledgement that they are often socially misunderstood.

Of course, this is not to say that autism and attachment must be unrelated. Indeed, it may well be that insecure attachments are sometimes the result of a parent's need for extra support when raising their autistic child (Schieve et al., 2007). Children with autism may also exhibit a multitude of behavioural or social difficulties, which may influence developing attachments with their parents (Rutgers et al., 2007). As such, given that these social and communication issues may overlap with insecure attachment styles, it is important to develop tools that disentangle these concepts before any associations between the two can be identified.

\section{Attachment interviews and autism}


Rather than administer questionnaires, researchers may utilise the Adult Attachment Interview (AAI; George et al., 1996). The AAI is an in-depth, hour-long interview with a researcher, carried out in order to assign an attachment style to the participant. It is a powerful assessment tool owing to its controlled protocol, assessment by trained researchers, and careful transcription of each spoken response in order to arrive at an attachment classification (Taylor et al., 2008). However, as noted by Taylor and colleagues, using this interview to assign attachment styles to those with autism can be problematic. The AAI, like most attachment questionnaires, was developed using a sample of neurotypical adults. The authors note that "differences in cognitive and verbal style might make them [those with autism] less able to talk coherently, relevantly and believably, or they may be more likely to be dismissive of discussing attachment relationships if they are aware of their own difficulties" (Taylor et al., 2008, p. 146). Although many adults with autism are able to discuss their experiences, the very nature of an interview as a social situation may prevent ease of communication in those with social and communication difficulties. Further, unless a researcher is trained in recognising and understanding the behaviours of adults with autism, those behaviours which are due to cognitive differences may be misattributed to insecure attachment styles in the same way that questionnaire responses might reflect physical discomfort or social rejection, rather than impairments in relationship attachment.

\section{Power and generalisability}

Power is an important issue in all research, particularly in the current climate in which psychological results have frequently failed to replicate. Studies which investigate autism and attachment fall into two broad categories, each with their own issues regarding power. 
First, studies such as Taylor et al. (2008) use the AAI to determine attachment styles, and compare groups based on the styles assigned to participants. As the AAI takes a significant amount of time to conduct, it is understandably difficult to assess attachment in large samples. However, researchers should always avoid drawing conclusions based upon underpowered samples. For instance, to detect a difference in the proportion of securely attached (versus other classifications) adults in autistic versus control samples with $80 \%$ power at an alpha of .05 , researchers would require around 390 participants in each group to detect a small effect. Even for a medium effect, 64 people are needed in each group, in comparison with 20 per group in Taylor et al. (2008).

Second, other studies, which may appear to be sufficiently powered, often use exclusively student populations as their participant pool. More broadly, their use as a proxy for the general public can be problematic (Hanel \& Vione, 2016). Here, researchers assume that associations between autistic traits and attachment in the population can be generalised to clinically diagnosed autistic individuals. Approximately $2 \%$ of undergraduates have clinically diagnosable autism (Macleod \& Green, 2009). Therefore, samples of 468 students (Takahashi et al., 2013), 326 (Gallitto \& Leth-Steensen, 2015), and 263 (Beffel et al., 2021) likely contain clinically diagnosable samples of fewer than ten people. As such, conclusions based upon almost entirely neurotypical populations may have limited applicability to autistic attachment styles.

Importantly, autism is further complicated by its broad and diverse constellation of traits, which incorporate different types of impairments that only sometimes co-occur (Ronald et al., 2005). Therefore, researchers must always keep in mind that generalisation to autistic populations as a whole based on clinically diagnosed subgroups may be problematic.

\section{Our recommendations}


Development of an autism-inclusive questionnaire

It is clear that there is a considerable need for an attachment measure that can be used by autistic adults, which takes into account the social and communication issues that may be present independent of any attachment-related issues. Of course, this may not be a simple task as there is still work to be done in understanding the interplay between autistic behaviours and their overlap with attachment styles. The goal must be to ensure that an adult with autism who has a secure attachment to their partner is not misclassified as insecure or uninvolved through the use of an instrument designed for neurotypical adults.

First, we must adjust our understanding of attachment styles in the context of autism, which may not reflect prototypical assumptions (based on neurotypical individuals). As noted above, "close" can be problematic for very different reasons when it comes to autistic attachment within relationships. Second, a measurement instrument should be developed which accounts for the communication issues that autistic individuals can face, and attachment should be explored and quantified while unrestricted by these barriers.

\section{Systematic reviews}

Across studies, measures of attachment and autism vary dramatically, making it difficult to draw any conclusions. In addition, we have highlighted here that measurement tools differ in their suitability for this task. An early meta-analysis with children (Rutgers et al., 2004) showed that those with autism were significantly less securely attached to their parents than controls but this was no longer the case when restricting samples to those with fewer autistic 
traits. Problematically, no recent reviews have weighed up contemporary evidence to determine the nature of this relationship in children or adults.

\section{An autism-inclusive Adult Attachment Interview}

Autism-inclusive attachment interviews with qualified clinicians who specialise in autism behaviours may be beneficial for understanding attachment in adults with autism. As the AAI was developed for neurotypical adults, the protocol naturally follows certain assumptions about attachment behaviours, and also about behaviours during the interview. Given the unique communication challenges that some adults with autism face, these individuals may be at a disadvantage whilst participating in interviews, and a well-meaning researcher who has little experience with autism may miscategorise autistic participants on the basis of a protocol which has not been designed for this population. As such, it is important that researchers develop an autism-inclusive protocol which accounts for the challenges that a participant with autism may face during the interview, but also that those conducting the interview are aware of the similarities between behaviours that represent insecure attachment and those which indicate autism.

\section{A note on attachment measures for children}

The issue of attachment measures is not unique to adults. A meta-analysis by Rutgers et al. (2004) showed that infants with autism are more likely to be classified as insecurely attached than neurotypical children. Further, they argue that this effect is more pronounced in children with a greater number of autistic traits. Could it be that autistic children simply do not communicate their needs in the same way as neurotypical children? The Strange Situation 
procedure (used to assess infant attachment) relies on social/communication behaviours as a measure of this attachment. It may be that a child with autism is distressed with the departure of their mother, and pleased upon her return, but simply cannot communicate this distress or pleasure in the same way as a typically developing child, thus earning them an 'insecure' classification. As research has shown, autistic children with fewer social difficulties may simply be better at communicating, or are more able to mask their autistic behaviours in order to gain a favourable response (for a review, see Allely, 2019). In this sense, the Strange Situation becomes a measure of how well the autistic child performs in the task, as opposed to a reflection of their true attachment to parents. That infants who later received an autism diagnosis were disproportionately more likely to be classified as insecure using the Strange Situation (Martin et al., 2020) begs the question as to whether this reflects actual attachment. Therefore, the limits of attachment measures for assessing those with autism may apply to both autistic children and adults alike.

\section{Conclusion}

The nature of attachment in individuals with autism is not well understood, but has important implications regarding long-term outcomes. Here, we discuss the difficulties with classifying attachment in this population, as well as the need to develop tools which are better suited to measuring attached in such clinical populations. Autism poses a particular problem for researchers due to the potential for autistic traits to be easily misconstrued as signs of an insecure attachment style. Therefore, we highlight the need to develop new tools that are specifically created in order to address this issue.

\section{References}


Allely, C. S. (2019). Understanding and recognising the female phenotype of autism spectrum disorder and the "camouflage" hypothesis: A systematic PRISMA review. Advances in Autism, 5(1), 14-37.

Baron-Cohen, S., Wheelwright, S., Skinner, R., Martin, J., \& Clubley, E. (2001). The Autism-Spectrum Quotient (AQ): Evidence from Asperger syndrome/high-functioning autism, males and females, scientists and mathematicians. Journal of Autism and Developmental Disorders, 31(1), 5-17.

Beffel, J. H., Cary, K. M., Nuttall, A. K., Chopik, W. J., \& Maas, M. K. (2021). Associations between the broad autism phenotype, adult attachment, and relationship satisfaction among emerging adults. Personality and Individual Differences, 168, 110409.

Brennan, K. A., Clark, C. L., \& Shaver, P. R. (1998). Self-report measurement of adult attachment: An integrative overview. In J. A. Simpson \& W. S. Rholes (Eds.), Attachment theory and close relationships (pp. 46-76). New York: Guilford.

Gallitto, E., \& Leth-Steensen, C. (2015). Autistic traits and adult attachment styles. Personality and Individual Differences, 79, 63-67.

George, C., Kaplan, N., \& Main, M. (1996). Adult Attachment Interview. Unpublished manuscript, Department of Psychology, University of California, Berkeley.

Hanel, P. H., \& Vione, K. C. (2016). Do student samples provide an accurate estimate of the general public? PLoS ONE, 11(12), e0168354.

Lau, W., \& Peterson, C. C. (2011). Adults and children with Asperger syndrome: Exploring adult attachment style, marital satisfaction and satisfaction with parenthood. Research in Autism Spectrum Disorders, 5(1), 392-399. 
MacLeod, A., \& Green, S. (2009). Beyond the books: Case study of a collaborative and holistic support model for university students with Asperger syndrome. Studies in Higher Education, 34(6), 631-646.

Martin, K. B., Haltigan, J. D., Ekas, N., Prince, E. B., \& Messinger, D. S. (2020). Attachment security differs by later autism spectrum disorder: A prospective study. Developmental Science, 23(5), e12953.

Ronald, A., Happé, F., \& Plomin, R. (2005). The genetic relationship between individual differences in social and nonsocial behaviours characteristic of autism. Developmental Science, 8(5), 444-458.

Rutgers, A. H., Bakermans-Kranenburg, M. J., van Ijzendoorn, M. H., \& van BerckelaerOnnes, I. A. (2004). Autism and attachment: A meta-analytic review. Journal of Child Psychology and Psychiatry, 45(6), 1123-1134.

Schieve, L. A., Blumberg, S. J., Rice, C., Visser, S. N., \& Boyle, C. (2007). The relationship between autism and parenting stress. Pediatrics, 119(Supplement 1), S114-S121.

Takahashi, J., Tamaki, K., \& Yamawaki, N. (2013). Autism spectrum, attachment styles, and social skills in university student. Creative Education, 4(08), 514-520.

Taylor, E. L., Target, M., \& Charman, T. (2008). Attachment in adults with high-functioning autism. Attachment \& Human Development, 10(2), 143-163. 